ment and is apt to tire after a 3 or 4 mile walk, but $I$ anticipate this will right itself in time. I must finish by saying that I should not have had the slightest chance of recovery had it not been for the patience and generosity shown to me by the surgeons who treated me, and for the kindness of numerous friends who looked after me during my somewhat horrible experience.

\title{
ENDOTHELIOMA OF THE SEROUS MEMBRANES IN THE OX.
}

\author{
By A. W. Trotter, M.R.C.V.S., Glasgow.
}

PATHOLOGISTS are not in unison as to whether endothelioma ought to be classed with the carcinomata or with the sarcomata. This form of neoplasm undoubtedly originates from non-epithelial structures, and it is upon this that those who claim that this neoplasm ought to be regarded as a sarcoma base their contention. On the other hand, as the histology of these neoplasms is similar to that of a hard cancer, others assert that they ought to be classified among the carcinomata. It may therefore be described as a hybrid neoplasm originating from tissues which ought to produce, according to pathological laws, a typical sarcoma, but which on the contrary presents on microscopical examination the characters of a cancer. The causation of endothelioma is, like all other forms of neoplasm, obscure, and much investigation is still required to elucidate its etiology.

It would appear that endothelioma of the serous membranes may occur in man, either as a large single growth or as small multiple diffuse growths. Senn, in his work on Pathology and Surgical Treatment of Tumours, p. 554, 2nd Edition, states: "The tumour may be nodular and of considerable size, or multiple," and quotes a case recorded by Nepvue, who described an endothelial sarcoma of the pleura in a child seven years of age. The tumour was the size of an adult's head, and displaced the lung.

The condition which forms the subject of this article differs from the case referred to above and conforms to that referred to by Ziegler in his Special Pathological Anatomy. At p. 875 this author states: "Endothelioma takes the form of diffuse white fibroid thickenings of the pleura, with a certain number of nodose swellings scattered through them; it may appear on any of the parietal, pulmonary, or diaphragmatic surfaces. Its coarse fibrous stroma encloses nests and reticulated strings of cells that give it an appearance not unlike that of a hard cancer." Again, p. 69I, "Endotheliomata (of the peritoneum) generally take the form of multiple flattened nodular growths, white in colour, and either coalescent or connected by neoplastic bands... The tumour is characterised by the presence in it of nests and clusters of endothelial cells, which lie in a dense fibrous stroma and follow exactly the course of the lymphatic vessels. They arise from the multiplication of the endothelial cells of the serous surface or of the lymphatics."

The translators of Kitt's Text-Book of Comparative General Pathology, in a note on p. 383 , state that "Endotheliomata of the 
pleura especially have been frequently described." They do not, however, state whether the cases referred to occurred in man or in the lower animals. Many cases have been placed on record of this condition having been found in man. For instance, Coats published in the Glasgow Medical Journal for July I 889 particulars of a case which came under his notice. On the pleura were numerous tumours and also, by coalescence, larger masses, forming a layer of considerable thickness. The tumours were almost continuous over the pleura, and there was no tumour anywhere else. He regarded it as a superficial cancer of the pleura.

In Friedberger \& Fröhner's Veterinary Pathology, p. 473, I898 Edition, it is stated that "sarcomatoses and carcinomatoses often assume in horses, and especially in dogs, the aspect of chronic peritonitis or pleuritis with abundant serous or hemorrhagic exudations." This part of their work is unfortunately extremely indefinite and unsatisfactory.

Contributions recording the occurrence of endotheliomata in bovines are very meagrely found in literature. It is not, however, an extremely rare condition. It has been recognised on many occasions during the last twelve years among bovines slaughtered for the food of man in the City abattoirs, Glasgow. Two cases were detected among 34,770 cattle slaughtered during the six months December I9I I to May 1912. This condition was in every instance found in aged cows, many of which were in fair condition and showed no evidence of ill health on ante-mortem examination. The following may be taken as typical of all cases which have come under my observation.

Subject.-An aged cross-bred cow in fair condition.

History.-She was bought from a cattle dealer at a country mart in Lanarkshire. Her previous history could not be ascertained. There were no manifest symptoms to indicate the presence of disease.

Post-mortem Examination.-On opening the abdominal cavity a large quantity of fluid escaped. The viscera, as they protruded through the opening, were seen to be studded with nodular growths.

Omentum.-The surface of the omentum was nodular and greyish in colour. It cut with a well-defined edge, and in places it was seen to have attained a thickness of $3 \mathrm{~cm}$. The nodules which had formed on each surface had by extension coalesced with their fellows on the opposite side, and had displaced the normal adipose tissue, which in places persisted merely as isolated areas. This condition was more pronounced in the vicinity of attachment to the rumen.

Intestines and Mesentery.--The surface of the intestines and mesentery was studded with nodules varying in size from a millet seed to a pea. At places the nodules had coalesced and had formed irregular areas.

Stomachs.-The mucous membranes were normal. The serous coats were studded with sharply defined nodules. These nodules were grey in colour, and varied in size from a millet seed to a pea. At places they had coalesced and had formed irregular areas. Some were firmly attached, but there was no evidence of their having penetrated to the muscular layer. They were more numerous in 
the vicinity of the attachment of the omentum. A few of the gastric lymph glands were larger and firmer than normal. On section, the lymphoid tissue in the vicinity of the afferent vessels was seen to have been replaced in a greater or less degree by fibrous tissue which gradually and imperceptibly blended with the normal tissue.

Spleen.-The surface of the spleen was studded with nodules varying in size from a pin head to a pea, and in colour from grey to red. Some were loosely, whilst others were firmly, attached to the capsule. The substance was normal.

Pancreas. - The substance of the pancreas showed areas varying from a millet seed to a pea in size, grey in colour, and firm in consistency. Their margin was sharply defined.

Liver. - The liver was normal in size. A few grey nodules varying from a millet seed to a pea were loosely adherent to Glisson's capsule. These nodules were more numerous in the vicinity of the attachment of the omentum, and, as a rule, were accommodated in small pits in the surface of the liver, formed through the parenchyma undergoing atrophy as the result of persistent pressure. The substance was normal. The hepatic group of ly mph glands was normal.

Kidneys.-The kidneys were normal. The renal lymph glands were normal.

Adrenals. - The adrenals were normal.

Uterus.-The serous coat of the uterus was nodular. The muscular and mucous coats were normal.

Ovaries.-The surface of the ovaries was nodular. The substance was normal.

Peritoneum.-The anterior half of the peritoneum was replaced by a thick fibrous layer, the surface of which was more or less distinctly nodular. Posteriorly this fibrous layer gradually became more and more nodular, until the colour of the underlying muscles was distinctly discernible between the nodules. The layer and nodules were grey in colour, firm in consistency, and cut with a well-defined edge. Both layer and nodules were firmly adherent to the underlying tissue, but there was no evidence, by macroscopical examination, of penetration. The cut surface was grey or greyish yellow, homogeneous, somewhat translucent, and revealed the presence of many minute tortuous blood vessels. The layer at its densest parts attained a thickness of $5 \mathrm{~cm}$.

Diaphragm.-The fibrous layer present on the anterior portions of the peritoneum was continued over the posterior surface of the diaphragm. On incising the muscles forming the "fleshy rim," particularly in the inferior third, dense white fibrous bands were observed to pass from the posterior to the anterior surface. These bands were undoubtedly lymphatic vessels, and were the means by which the disease had spread from the peritoneum to the pleural cavity. The anterior surface of the "fleshy rim," crura, and tendinous portions of the diaphragm were also affected, but in a lesser degree.

Pleura.-That part of the pleura covering the triangularis sterni was replaced by a fibrous layer similar in character to that present on the anterior portion of the peritoneum. This layer passed anteriorly, involved the pericardium, and was continued along the walls of the vessels as they entered the thoracic cavity between 
the first ribs. The margin of the layer was irregular, and superiorly it was prolonged upwards by a more or less continuous layer of nodules. These nodules were confined to the anterior and posterior edges of the ribs. They were, like the fibrous layer, firmly adherent to the underlying tissue. An examination of these nodules showed that in some fibrous bands were given off, which gave them the appearance of grotesque insects. These bands passed in some instances from one nodule to another, and exemplified the manner by which the fibrous layer was developed.

Lungs. - The surface of the lungs, particularly of those parts in contact with the diaphragm, the mediastinum, and the triangularis sterni, showed numerous nodules, which were almost invariably connected by thin bands of tissue presenting the same characters. These nodules and bands ran along the course of the interlobular septa. From some of the nodules branches were given off, and these became adherent to adjacent pulmonary tissue, and through contraction had caused in places puckering of the surface. At a few places these bands had penetrated into the substance of the lungs along the course of the interlobular septa. The posterior mediastinal lymph glands were enlarged, firm, and fibrous.

Heart.- The heart was normal.

Mammary Glands.-The mammary glands and the supra-mammary lymphatic glands were normal.

Trunk Lymphatic Glands.-The following groups of lymphatic glands were found normal: Lumbar, internal and external iliac, popliteal, precrural, brachial, prescapular, and dorso-costal. The following groups were affected: Supra-sternals, prepectorals, and those embedded in the fat lying superior to the ensiform cartilage and anterior to the diaphragm.

In some of my cases the dense layer present on the peritoneum was on incision found to have undergone extensive mucoid degeneration. The layer frequently attained a thickness of $3 \mathrm{~cm}$., with the central portions almost wholly occupied by a trembling jelly-like substance of a reddish tint.

Microscopical Examination.-On microscopical examination the histology of an endothelioma is seen to be analogous to that of a carcinomata in that there is a fibrous stroma and cells possessing the characters of epithelium. The latter are sharply demarcated from the former, and are so arranged as to present an interminable variety of shape and size; in some cases they are in oval, pyriform, oblong, or irregular masses, whilst others are in straight or tortuous lines. The arrangement of the cells depends largely on the angle at which the section is cut, as in places they are in a single layer arranged in circular formation, in others in two parallel rows, which are in many cases separated by a central space corresponding to the lumen of a vessel. It is therefore evident that this condition has originated in the lymph system. The cells in these positions are cubical in shape. It sometimes happens, however, that owing to proliferation the cells are in masses. In these cases the cells assume a variety of shapes. The stroma may either be scanty or abundant. In places the masses or lines of cells are separated only by a small amount of fibrous tissue, but the latter, as the disease progresses, becomes more abundant. The cells as the result of pressure undergo 
degeneration, and ultimately disappear. All phases of this gradual transformation may be discerned from the area showing merely a dense cellular infiltration, the invasion of adjacent tissue by these round cells or fibroblasts, and their development into mature fibres.

Abnormal lymph capillaries may be seen penetrating the deeper tissues, demonstrating that this condition is not limited to the surface layers, as would appear on macroscopical examination.

\title{
PERIODONTAL DISEASE IN THE HORSE,
}

\author{
By W. L. LiTTLE, F.R.C.V.S., Yarmouth.
}

THE form of periodontal disease, or alveolar periostitis, to which these notes refer is that which is most frequently found in the horse. It is peculiar to that species, and causes them to drop their food in a characteristic manner, known by some as "quidding."

It starts primarily in the spaces between the cheek teeth, usually of the lower jaw, and more often affects the second molar than any other; the premolars are not often affected, and when they are they do not seem to cause so much trouble as the molars.

It is found that the farther back in the mouth the diseased condition exists the more exaggerated are the symptoms shown. This is probably because in the act of mastication more pressure is brought to bear on the molars than the premolars.

When a premolar is affected it is often found that a molar is also, and as a general rule both sides of the mouth are affected at the same time.

It is found in horses over four years of age and all breeds. Heavy horses are perhaps more liable than the lighter varieties, and those of some districts more so than others; in some districts I am told it is almost unknown, while in others it is very prevalent.

I have operated upon or assisted in operating upon an average of fifteen horses each year for the past ten years. This of course does not include every case which I have detected.

Mr I. F. Colyer, M.R.C.S., in the Veterinary Record, 23rd September I905, says that out of 500 skulls of horses that died in London 166 showed signs of this disease. I should think several of these cases were not sufficiently advanced to cause much difficulty in feeding, and were probably aged horses, which nearly always show this disease in a slight form.

Normally the spaces between the teeth are hardly appreciable. When these spaces are larger than normal particles of food accumulate in them, this being favoured by the shape of the teeth. The horse's tongue not being adapted for the removal of the material, the accumulation continues, and the act of mastication forces it into a compact mass or small "quid." This presses on the alveolar rim, and, aided by its decomposition, sets up inflammation of the mucous membrane of the gum, which spreads to the periodontal membrane.

If this small quid remains, as it will do if not interfered with from outside, the space gradually becomes larger until it forms what may 